\title{
Inhibitory Effects of Ketoconazole, Cimetidine and Erythromycin on Hepatic CYP3A Activities in Cats
}

\author{
Syed Sher SHAH ${ }^{1}$, Kazuaki SASAKI $^{1}$, Yuhei HAYASHI ${ }^{1}$, Seiko MOTOYAMA ${ }^{1}$, Abdou Rania HELMI ${ }^{1}$, \\ Waleed Fathy KHALIL ${ }^{1)}$ and Minoru SHIMODA ${ }^{1) *}$ \\ ${ }^{1)}$ Department of Veterinary Medicine, Tokyo University of Agriculture and Technology, Saiwai-cho 3-5-8, Fuchu, Tokyo 183-8509, Japan
}

(Received 16 February 2009/Accepted 1 May 2009)

ABSTRACT. Inhibitory effects of ketoconazole (KTZ), cimetidine (CIM), and erythromycin (ERY) were examined on CYP3A activities. Midazolam 1'- and 4-hydroxylation (MDZ1'H and MDZ4H) were used to determine CYP3A activities in hepatic microsomes obtained from cats $(n=4)$. The results showed that, all the examined drugs inhibited the reactions in a noncompetitive manner. The inhibitory constants $(\mathrm{Ki})$ of $\mathrm{KTZ}$ were $2.80 \pm 0.70$ and $115 \pm 28 \mu \mathrm{M}$ for MDZ1' $\mathrm{H}$ and MDZ4H, respectively. Those of CIM were 3.13 and 3.27 $\mathrm{mM}$ and of ERY were 3.14 and $6.41 \mathrm{mM}$ for MDZ1'H and MDZ4H, respectively. Mechanism-based inhibition was also examined in this study. KTZ significantly reduced MDZ reactions in a time-dependent manner; while CIM and ERY did not. Also, the effects of KTZ and CIM on the pharmacokinetics of quinidine (QUN) were studied. KTZ or CIM $(10 \mathrm{mg} / \mathrm{kg} / \mathrm{day}$, for one week) was given orally to cats $(\mathrm{n}=5)$. QUN (2 mg/kg, i. v.) was injected $2 \mathrm{hr}$ after the last dose of KTZ or CIM. The analysis of the obtained pharmacokinetic parameters showed that, KTZ significantly reduced total body clearance of QUN by $35 \%$, while CIM did not. These results suggest that, KTZ inhibits CYP3A activities (both in vitro and in vivo), but CIM does not. In clinical practice, therefore, KTZ may result in inhibition based drug-drug interaction with CYP3A substrates in cat patients, whereas CIM and ERY are unlikely to lead to interaction involving CYP3A substrates.

KEY WORDS: cimetidine, CYP3A, enzyme inhibition, feline, ketoconazole.

J. Vet. Med. Sci. 71(9): 1151-1159, 2009

The cytochrome P450 (CYP)-mediated drug metabolism is one of most important elimination pass ways in drug elimination. Of the CYP enzymes, CYP3A is the most important subfamily in human, because it catalyses biotransformation of approximately half of currently available drugs in clinical practice $[1,8]$. The reduction in CYP3A activities, therefore, may result in serious adverse effects in drug therapy, because of increased bioavailability and decreased clearance of drugs.

Consequently, many researchers extensively studied the possible inhibitory effects of drugs on CYP3A activities $[11,25,27]$. As a result, many drugs were classified as a CYP3A inhibitor, including azole antifungal agents $[17,26$, 32], cimetidine (CIM) [4, 9, 15], and macrolide antibiotics [32] in humans or dogs. Many investigators have also demonstrated drug-drug interaction between CYP3A inhibitors and CYP3A substrates. In human, Yamano et al. [30] reported increasing plasma concentrations of midazolam (MDZ) with concomitant administration of CIM, itraconazole or erythromycin (ERY). They also reported that CIM, itraconazole and ERY decreased hepatic intrinsic clearance of MDZ by $30 \%, 60 \%$, and $80 \%$, and hepatic clearance by $20 \%, 50 \%$, and $70 \%$, respectively. In dogs concomitant use of ketoconazole (KTZ) decreased the total body clearance (Cltot) of nifedipine by $71 \%$ [10], that of MDZ by $71 \%$ [11], and that of quinidine (QUN) by about $60 \%$ [13].

\footnotetext{
* Correspondence to: Shimoda, M., Department of Veterinary Medicine, Tokyo University of Agriculture and Technology, Saiwai-cho 3-5-8, Fuchu, Tokyo 183-8509, Japan. e-mail :ms@ cc.tuat.ac.jp
}

Although it is well known that cats have quite low or negligible activities of UDP-glucuronosyltransferase, information about CYP3A-mediated drug metabolism is limited in cats. However, Shah et al. [22] have recently reported that 1 '- and 4-hydroxylation of MDZ is mainly catalysed by CYP3A also in cats. Based on intrinsic clearance of the reaction, they suggested that CYP3A activities in female cats are similar to those in humans but lower than those of dogs. They also found that, these activities in male cats are higher than female cats and humans activities but similar to those in dogs [11].

Multiple drug therapy is frequently applied to cat patients. In addition, cat patients are often prescribed drugs that are used in human medicine. Of these drugs, some have been demonstrated to be CYP inhibitors in human. Therefore, there are possibilities of drug-drug interaction also in cats. Veterinary practitioners, therefore, should pay much attention to such drug-drug interaction. However, there are only a few reports describing potential drug-drug interaction in cats [23].

Although KTZ, CIM, and ERY were reported as CYP3A inhibitors in human, they are extensively used in many countries for treatment of cats without any restrictions due to lack of information about effects of these drugs on CYP enzyme activities. In the present study, therefore, we examined the inhibitory effects of KTZ, CIM, and ERY on CYP3A activities using feline hepatic microsomes. We also examined effects of multiple oral doses of KTZ and CIM on pharmacokinetics of QUN. 


\section{MATERIALS AND METHODS}

Chemicals: MDZ, 1'-hydroxymidazolam and 4hydroxymidazolam were purchased from Daiichi Chemicals Co., Ltd (Tokyo, Japan). Glucose-6-phosphate, glucose-6phosphate dehydrogenase, and ERY were obtained from Sigma Chemical Co. (St. Louis, Mo, U.S.A.). KTZ, quinidine sulfate and CIM were purchased from Wako Pure Chemical Co., Ltd. (Osaka, Japan).

Animals: Nine female cats (short hair, 1-2.5 years old, weighing 2.2 to $3.0 \mathrm{~kg}$ ) were obtained from Iffa Credo (France). Each cat was housed separately in a stainless-steel cage with a 12-hr light/dark cycle. Stable temperature and relative humidity were maintained at $19-22^{\circ} \mathrm{C}$ and $40-70 \%$, respectively. The cats were given food (Science diet, feline maintenance, Hill's pet Nutrition, Topeca, KS, U.S.A.) once a day and allowed access to water ad libitum. The experiments in this study were conducted in accordance with the guidelines for the care and use of laboratory animals and approved by the committee of animal experiment, Tokyo University of Agriculture and Technology.

Preparation of feline liver microsomes: Four cats were deprived food overnight before the experiment. They were deeply anesthetised by intravenous injection of pentobarbital sodium (Nembutal ${ }^{\circledR}$; Dainippon Pharmaceutical Co., Ltd, Osaka, Japan) at a dose of $25 \mathrm{mg} / \mathrm{kg}$ body wt., exsanguinated from a cannula inserted into the carotid, and then the liver was immediately isolated. The liver was instantly perfused with an ice-cold buffer $(1.15 \% \mathrm{KCl}, 0.2 \mathrm{mM}$ EDTA-2Na, $0.1 \mathrm{mM}$ dithiothreitol, $0.1 \mathrm{mM}$ phenyl methyl sulphonyl fluoride, and $20 \%$ glycerol) from the caudal vena cava until the efflux perfusion buffer was blood free, and then stored at $-80^{\circ} \mathrm{C}$ until preparation of microsomes.

Microsomal fractions were prepared from the liver specimens as described by van der Hoeven and Coon [24]. The obtained microsomal suspension was stored at $-80^{\circ} \mathrm{C}$ until used. The protein concentrations were determined by the method of Bradford [2] using a commercially available dye reagent (Bio-Rad Protein Assay ${ }^{\circledR}$, Bio-Rad Laboratories, Inc., CA, U.S.A.). CYP content was determined by the method described by Omura and Sato [18]. The obtained values were comparable to those described previously [22].

Enzyme assay: The reactions of MDZ 1'-hydroxylation $\left(\mathrm{MDZ1}{ }^{\prime} \mathrm{H}\right)$ and 4-hydroxylation $(\mathrm{MDZ} 4 \mathrm{H})$ were used to determine the CYP3A activities in the microsomes, as these reactions are mainly catalysed by CYP3A in cats [22]. The reaction proceeded at $37^{\circ} \mathrm{C}$ in $50 \mathrm{mM}$ phosphate buffer $(\mathrm{pH}$ 7.4) containing NADPH-generating system $(0.5 \mathrm{mM}$ $\mathrm{bNADP}^{+}, 5 \mathrm{mM}$ glucose-6-phosphate, $1.5 \mathrm{U} / \mathrm{m} l$ glucose-6phosphate dehydrogenase, and $5 \mathrm{mM} \mathrm{MgCl}_{2}$ ) and $0.3-0.5$ $\mathrm{mg}$ microsomal protein in a total assay volume of $0.25 \mathrm{ml}$. There was a $5 \mathrm{~min}$ preincubation at $37^{\circ} \mathrm{C}$ before the reaction was started by the addition of MDZ. The concentrations of MDZ in the assay system ranged from 15 to $307 \mu \mathrm{M}$. The reaction proceeded at $37^{\circ} \mathrm{C}$ for $10 \mathrm{~min}$ and stopped by adding $0.25 \mathrm{~m} l$ of acetonitrile. The sample was placed on ice for $5 \mathrm{~min}$ and then centrifuged at $10,000 \mathrm{~g}$ for $2 \mathrm{~min}$. The supernatant was filtered with a $0.45 \mu \mathrm{m}$ filter (Chromatodisk 4P, Biofield Co., Ltd., Tokyo, Japan), and $50 \mu l$ of the filtrate was immediately analysed to determine the concentrations of MDZ metabolites.

Reversible inhibition test: KTZ, CIM and ERY were dissolved in ethanol, and $10 \mu l$ of the solution was added in the assay system just before the addition of MDZ. The concentration of KTZ was $1 \mu \mathrm{M}$ for MDZ1'H and $0.1 \mathrm{mM}$ for MDZ4H in the assay system. The concentrations of CIM and ERY were 2.4 and $10.9 \mathrm{mM}$, respectively, in the assay system.

Mechanism-based inhibition test: After the preincubation of the assay system for $5 \mathrm{~min}$, each inhibitor was added, and then the sample was incubated for $0,10,20$ or 30 min before substrate was added at a concentration of $123 \mu \mathrm{M}$. The concentrations of each inhibitor in the assay system were the same as those in the reversible inhibition test.

Determination of MDZ metabolites: The hydroxylated metabolites of midazolam were determined using a reversed phase HPLC with UV-detection as described by Kuroha et al. [12]. The analytical column was $\mathrm{C}_{18}$ column (TSK-gel ${ }^{\circledR}$; ODS-120T, $5 \mu \mathrm{m}$ particle size, $250 \times 4.6-\mathrm{mm}$ i.d., Tosoh Co., Tokyo, Japan). Column effluent was monitored at 254 $\mathrm{nm}$ wavelength using an UV-detector (SPD-6A, Shimadzu Corporation, Kyoto, Japan). The mobile phase was a mixture of $100 \mathrm{mM}$ acetate buffer $(\mathrm{pH} \mathrm{4.7)}$, acetonitrile and methanol $(59.4: 35: 5.6, \mathrm{v} / \mathrm{v} / \mathrm{v})$. The flow rate of the mobile phase was $1 \mathrm{ml} / \mathrm{min}$. The detection limits of 1'- and 4hydroxymidazolam were 3.6 and $2.5 \mathrm{ng} / \mathrm{ml}$, respectively, at a signal-to-noise ratio of 3 . The recovery of 1'-hydroxymidazolam was $101 \pm 1.4 \%(\mathrm{CV}=1.2 \%)$ at $1 \mu \mathrm{g} / \mathrm{m} l(\mathrm{n}=4)$. The intra-day $\mathrm{CV}$ values were $1.4 \%$ and $5.4 \%$ at 0.1 and $1 \mu \mathrm{g} / \mathrm{ml}$, respectively $(\mathrm{n}=4)$. The inter-day $\mathrm{CV}$ values ranged from $1.2 \%$ to $2.4 \%$ and from $2.1 \%$ to $5.7 \%$ at 0.1 and $1 \mu \mathrm{g} / \mathrm{ml}$, respectively ( 3 days, 4 determinations/day). The recovery of 4-hydroxymidazolam was $102 \pm 3.4 \%(\mathrm{CV}=2.4 \%)$ at 1 $\mu \mathrm{g} / \mathrm{m} l(\mathrm{n}=4)$. The intra-day $\mathrm{CV}$ values were $1 \%$ and $3.9 \%$ at 0.1 and $1 \mu \mathrm{g} / \mathrm{m} l$, respectively $(\mathrm{n}=4)$. The inter-day $\mathrm{CV}$ values ranged from $0.2 \%$ to $0.5 \%$ and from $2.6 \%$ to $8.5 \%$ at 0.1 and $1 \mu \mathrm{g} / \mathrm{ml}$, respectively ( 3 days, 4 determinations/day).

Enzyme kinetic analysis: Lineweaver-Burk plots were used to determine the type of inhibition by the inhibitors and to obtain initial values for a nonlinear least squares regression. Since the plots suggested noncompetitive inhibition for each inhibitor, the following equations were used to analyse the enzyme kinetics of MDZ4H in absence or existence of inhibitors,

$$
\begin{gathered}
v=\frac{V_{\max } \times S}{K_{m}+S} \\
v=\frac{V_{\max } \times S}{\left(K_{m}+S\right)\left(1+\frac{I}{K_{i}}\right)}
\end{gathered}
$$


Where $V_{\max }$, and $K_{m}$ represent maximal reaction velocity and Michaelis-Menten constant, respectively. S and I are concentrations of the substrate and inhibitors, respectively. $K_{i}$ is the inhibitory constant (dissociation constant of inhibitors). The kinetic profile of MDZ1'H was consistent with Michaelis-Menten kinetics with uncompetitive substrate inhibition [11], and therefore the following equations were fitted to MDZ1' $\mathrm{H}$ kinetics in absence or existence of inhibitors,

$$
\begin{aligned}
& v=\frac{V_{\max } \times S}{K_{m}+S\left(1+\frac{S}{K_{s}}\right)} \\
& v=\frac{V_{\text {max }} \times S}{\left(1+\frac{I}{K_{i}}\right)\left(K_{m}+S\left(1+\frac{S}{K_{s}}\right)\right)}
\end{aligned}
$$

Where $K_{s}$ represents substrate inhibition constant.

Two curves, which were obtained with or without inhibitors in each reaction, were simultaneously analysed by use of the fitting program MULTI [29] to calculate $V_{\max }, K_{m}$ and $K_{i}$ for MDZ4H and these parameters and Ks. for MDZ1'H.

\section{Effect on QUN pharmacokinetics}

Study design: In this study, the inhibitory effects of one week treatment of KTZ and CIM on pharmacokinetics of QUN were examined in five cats. QUN was chosen as a CYP3A substrate because it is one of CYP3A substrates in other animal species [13] and showed low hepatic extraction profiles in cats in preliminary experiments. For control, QUN was intravenously injected at $2 \mathrm{mg} / \mathrm{kg} 4$ weeks before the start of KTZ or CIM treatment. KTZ tablets (Nizoral ${ }^{\circledR}$ ) were obtained from Jansen Pharmaceutica (Titusville, NJ), and CIM tablets (Tagamet ${ }^{\circledR}$ ) from Dainippon Sumitomo Pharma (Osaka, Japan). These tablets were grinded into small pieces, weighed, and then orally administered to cats. $\mathrm{KTZ}$ was administered at $10 \mathrm{mg} / \mathrm{kg}$ just before feeding once daily. CIM was administered three times a day for a week, respectively. After $2 \mathrm{hr}$ of the final dose of the inhibitors, QUN was intravenously injected at $2 \mathrm{mg} / \mathrm{kg}$. There was a 4 weeks interval between KTZ and CIM treatment. QUN $(250 \mathrm{mg}$ ) was dissolved in $1.5 \mathrm{ml}$ ethanol. Then the final volume was adjusted to $50 \mathrm{~m} l$ by adding sterilized distilled water to yield $5 \mathrm{mg} / \mathrm{m} l$. The QUN solution was infused into the left cephalic vein at the rate of $0.1 \mathrm{~m} / / \mathrm{sec}$. About $1.5 \mathrm{ml}$ blood samples were collected from the right cephalic vein at $0.5,1,2,3,4,6$, and $8 \mathrm{hr}$ after QUN administration. The plasma was separated by centrifugation $(1,500 \mathrm{~g}, 5 \mathrm{~min})$ within $30 \mathrm{~min}$ from blood collection and stored at $-80^{\circ} \mathrm{C}$ until assayed. One day before the start of each experiment, blood samples were collected from each cat to confirm the absence of interfering peaks in the plasma.

Determination of plasma QUN concentrations: Plasma concentrations of QUN were determined by the HPLC with a fluorometric detector (RF-535; Shimadazu Corporation) as described by Edstein et al. [6] with slight modifications. After thawing, $0.2 \mathrm{~m} l$ plasma was spiked with $0.8 \mathrm{~m} l$ acetonitrile. The mixture was vortexed for a few seconds and then centrifuged $(16,000 \mathrm{~g}, 3 \mathrm{~min})$. The supernatant $(0.5 \mathrm{ml})$ was transferred to a clean pear-shaped flask and evaporated at $40^{\circ} \mathrm{C}$ to dryness under a reduced pressure using a rotary evaporator (Rotavapor ${ }^{\circledR}$, R-114, Shibata Scientific Technology Ltd. Tokyo, Japan). The residue was reconstituted in $0.5 \mathrm{ml}$ mobile phase. After filtration with the $0.45 \mu \mathrm{m}$ filter, $50 \mu l$ of the filtrate was injected into an HPLC $\mathrm{C}_{8}$ column $\left(\right.$ LiChroCART $^{\circledR}, 5 \mu \mathrm{m}$ particle size, $250 \mathrm{~mm} \times 4.6 \mathrm{~mm}$ i. d., Kanto Chemical Co., Inc., Tokyo, Japan). Excitation and emission wavelengths were 340 and $425 \mathrm{~nm}$, respectively. The mobile phase was a mixture of $0.4 \%$ triethylamine $(\mathrm{pH}$ 2.5 , adjusted with $7 \mathrm{M}$ phosphoric acid) and acetonitrile $(88: 12, \mathrm{v} / \mathrm{v})$, flow rate was $1 \mathrm{ml} / \mathrm{min}$. The detection limit was $1 \mathrm{ng} / \mathrm{ml}$ at a signal-to-noise ratio of 3 . The recovery of QUN was $97.7 \pm 3.4 \%(\mathrm{n}=5)$ at $1 \mu \mathrm{g} / \mathrm{m} l$. Intra-day $\mathrm{CV}$ values were $2.8 \%$ and $3.0 \%$ at 0.1 and $1 \mu \mathrm{g} / \mathrm{ml}$, respectively. The inter-day CV values ranged from $2.2 \%$ to $3.4 \%$ and $1.9 \%$ to $3.5 \%$ at 0.1 and $1 \mu \mathrm{g} / \mathrm{ml}$, respectively ( 3 days, 5 determinations/day).

Pharmacokinetic analysis: One compartment open model was used to analyse the pharmacokinetics of QUN. The plasma concentration at time $0 \mathrm{hr}\left(C_{p(0)}\right)$ and elimination rate constant $\left(K_{e l}\right)$ in the following equation were calculated using the nonlinear fitting program, MULTI [29],

$$
C p=C p_{(0)} e^{-K_{e l t}}
$$

where $C p$ and $t$ represent plasma concentration and time after QUN administration, respectively.

The area under the plasma concentration-time curve $(A U C)$ and of its first moment curve $(A U M C)$ were obtained as a sum of the area from 0 to last sampling time by trapezoidal method and that from the last sampling time to infinity by integration. The elimination half-life $\left(t_{1 / 2}\right)$, apparent volume of distribution $\left(V_{d}\right)$, total body clearance $\left(C l_{\text {tot }}\right)$ and mean residence time $(M R T)$ were calculated using the following equations,

$$
\begin{aligned}
& t_{1 / 2}=\frac{0.693}{K_{e l}} \\
& V_{d}=\frac{\text { Dose }}{C p_{(0)}} \\
& C l_{\text {tot }}=\frac{\text { Dose }}{\text { AUC }} \\
& M R T=\frac{A U M C}{A U C}
\end{aligned}
$$

Statistical analysis: When mean values from 2 groups were compared (in vitro results), the statistical significance 
was tested using a 2-tailed paired $t$-test. In case of more than 3 groups (pharmacokinetic results), it was tested using a repeated one-way ANOVA and Sheffe's multiple comparisons as a post-hoc test. The difference was considered significant when $\mathrm{P}<0.05$.

\section{RESULTS}

\section{Effect on MDZ-hydroxylation}

Reversible Inhibition: Representative of LineweaverBurk plots of MDZ4H is shown in Fig. 1. The plots indicated that KTZ, CIM and ERY inhibited the reaction by a noncompetitive manner, because 2 lines from with or without inhibitor crossed on the X-axis. Since MDZ1'H was inhibited by MDZ itself as shown in Fig. 2, we could not demonstrate the inhibitory mode of the inhibitors by Lineweaver-Burk plots. However we assumed noncompetitive inhibition to calculated $\mathrm{Ki}$ value, because the curves in Fig. 2 suggested that the inhibitors decreased $V_{\max }$. The theoretical lines calculated using enzyme kinetic parameters of Table 1 and equations 3 and 4 fitted well with the observed data (Fig. 2), suggesting that the KTZ, CIM and ERY noncompetitively inhibited MDZ1'H.

As shown in Table 1, KTZ had the smallest Ki value compared with CIM and ERY, indicating its inhibitory effect is the most potent among the examined drugs in this study. However, the extent of inhibitions on MDZ1' $\mathrm{H}$ and
MDZ4H were quite different. The $K_{i}$ value of $K T Z$ for MDZ4H was 40-fold higher compared with that for MDZ1'H. In contrast, the mean $K_{i}$ values of CIM and ERY were quite high and in the millimolar range.

Irreversible inhibition (mechanism-based inhibition): Figure 3 shows the effect of exposure time with KTZ, CIM, and ERY on MDZ1'H and MDZ4H. In case of KTZ, the reaction velocity significantly decreased by 10,20 and 30 min exposure, suggesting that KTZ inhibited the enzyme reactions by mechanism-based inhibition. Whereas in the case of ERY, the decreases were small even though high concentrations were used. Compared with KTZ, CIM showed similar decreases in the reaction, but its concentration was quite high.

Effect on QUN pharmacokinetics: The multiple oral treatment of KTZ (10 mg/kg, once daily for a week) altered the pharmacokinetics of QUN which was concomitantly administered (Fig. 4). As shown in Table 2, AUC increased by about 1.6 fold (1.962 to 3.158 ), elimination $\mathrm{t} 1 / 2$ was prolonged by about 1.6 fold ( 1.50 to $2.35 \mathrm{hr}$ ), and the $C l_{\text {tot }}$ decreased by $35 \%$ (1.031 to $0.673 \mathrm{~L} / \mathrm{h} / \mathrm{kg})$. These changes were statistically significant $(\mathrm{p}<0.05)$. On the other hand, the oral treatment of CIM $(10 \mathrm{mg} / \mathrm{kg}$, three times a day for a week) altered neither plasma concentration-time profiles (Fig. 4) nor the pharmacokinetic parameters of QUN (Table 2).

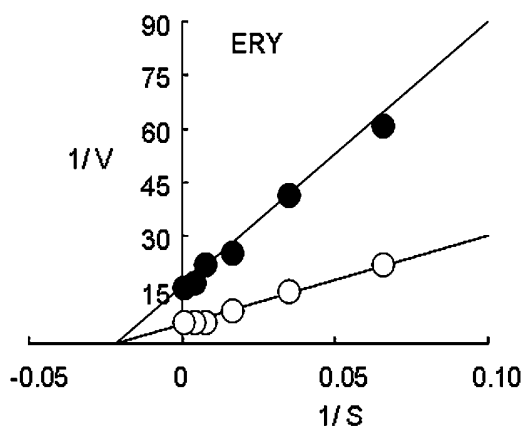

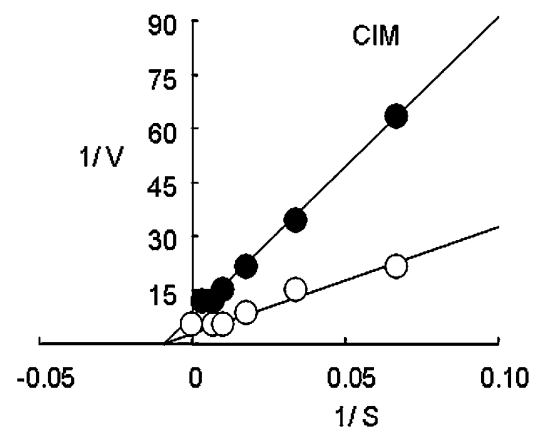

Fig. 1. Lineweaver-Burk plots of midazolam 4-hydroxylation (MDZ4H) with or without ketoconazole (KTZ), cimetidine (CIM) and erythromycin (ERY). Representatives from 4 cat microsomes are shown. Closed and open circles indicate the reactions with and without inhibitors, respectively. The units of substrate (S) and reaction velocity (V) are $\mu \mathrm{M}$ and $\mathrm{nmol} / \mathrm{min} / \mathrm{mg}$ protein, respectively. 

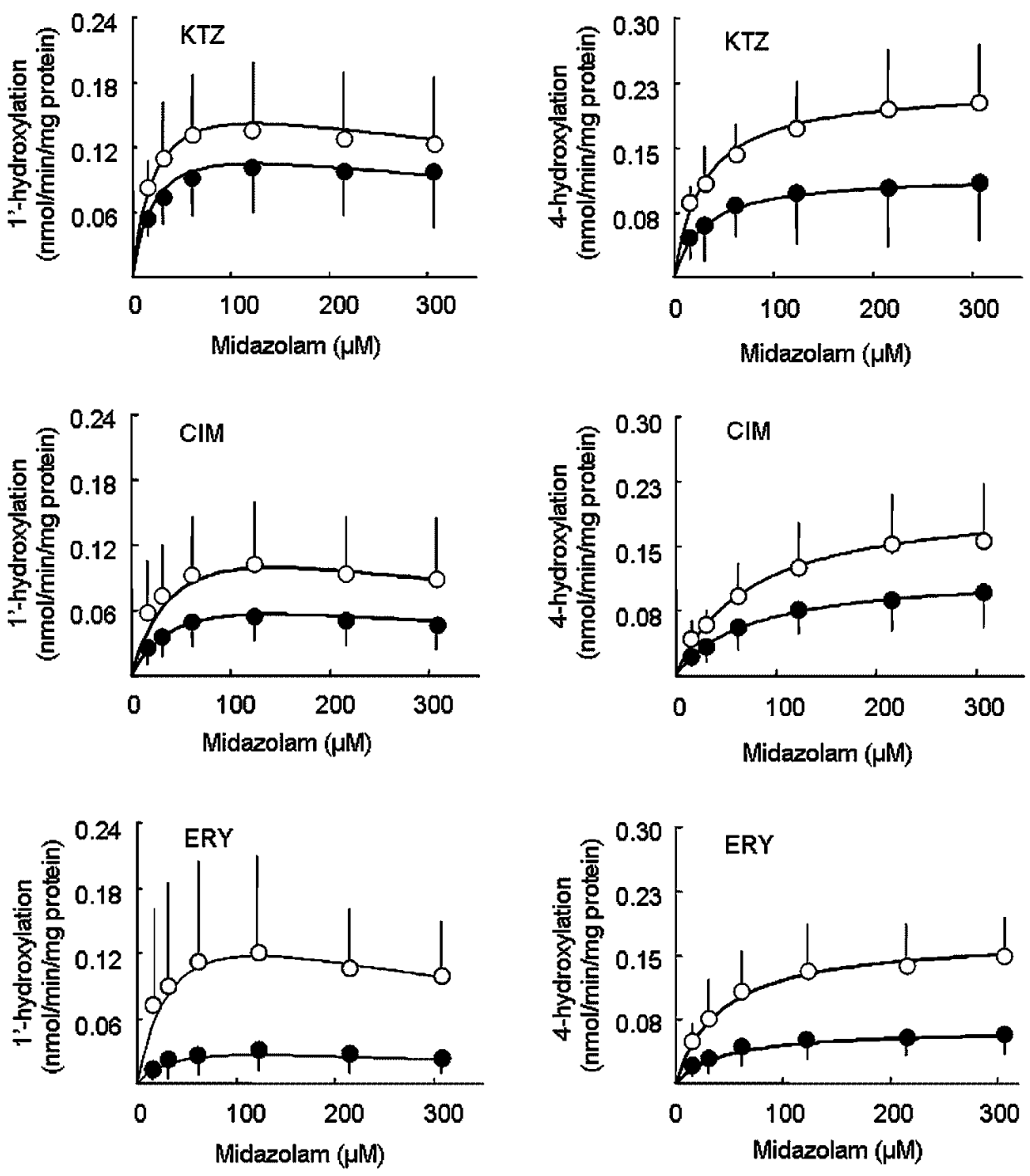

Fig. 2. Michaels-Menten kinetics of 1'- and 4-hydroxylation of midazolam with or without ketoconazole (KTZ), cimetidine (CIM) and erythromycin (ERY). Each point and vertical bar is represented by mean \pm SD from four microsomes. Open circles represent reactions without inhibitors. Closed circles represent the reactions with KTZ (1 $\mu \mathrm{M}$ for 1'-hydroxylation and $100 \mu \mathrm{M}$ for 4-hydroxylation), CIM (2.4 mM) or ERY $(10.9 \mathrm{mM})$. The concentrations of midazolam ranged from 15 to $307 \mu \mathrm{M}$. The solid curves in the figure were calculated by using equations 1 and 2 for 4-hydroxylation, equations 3 and 4 for 1'-hydroxylation in Materials and Methods section and enzyme kinetic parameters in Table 1.

\section{DISCUSSION}

In the present study, KTZ, CIM, and ERY inhibited both MDZ1'fH and MDZ4H catalysed by CYP3A in a noncompetitive manner in cat hepatic microsomes. Competitive inhibitions of these inhibitors have also been described in other animal species [12, 25, 28]. MDZ hydroxylation was most potently inhibited by KTZ with $K_{i}$ values of $2.8 \mu \mathrm{M}$ for MDZ1' $\mathrm{H}$ and $115 \mu \mathrm{M}$ for MDZ4H. On the other hand, $K_{i}$ values of CIM and ERY were quite higher for both MDZ1'H and MDZ4H reactions. Based on $K_{i}$ values, the inhibitory effects of KTZ may be substantial but those of CIM and ERY may not be substantial in clinical conditions. It is, therefore, suggested that concomitant administration of CYP3A substrate drugs with KTZ may result in drug-drug interaction in clinical condition in cats as in humans and dogs but CIM and ERY may not. McAnulty et al. [16] have reported that KTZ resulted in twice elevation in serum concentrations and about $60 \%$ decrease in the mean systemic clearance of cyclosporine in cats with renal transplantation. This finding may support our suggestion. Moreover, other researchers also reported drug interaction of KTZ with 
Table 1. Michaelis-Menten kinetic parameters for midazolam hydroxylation in presence or absence of ketoconazole, cimetidine and erythromycin in hepatic microsomes from cats

\begin{tabular}{lccccccc}
\hline & \multicolumn{3}{c}{1 '-hydroxymidazolam } & & \multicolumn{3}{c}{ 4-hydroxymidazolam } \\
\cline { 2 - 4 } Inhibitors & $\begin{array}{c}V_{\max } \\
(\mathrm{nmol} / \mathrm{min} / \mathrm{kg})\end{array}$ & $\begin{array}{c}K_{m} \\
(\mu \mathrm{M})\end{array}$ & $\begin{array}{c}K_{i} \\
(\mathrm{mM})\end{array}$ & & $\begin{array}{c}V_{\max } \\
(\mathrm{nmol} / \mathrm{min} / \mathrm{kg})\end{array}$ & $\begin{array}{c}K_{m} \\
(\mu \mathrm{M})\end{array}$ & $\begin{array}{c}K_{i} \\
(\mathrm{mM})\end{array}$ \\
\hline KTZ & $0.19 \pm 0.09$ & $22.74 \pm 9.21$ & $0.0028 \pm 0.0007$ & & $0.22 \pm 0.07$ & $29.38 \pm 5.90$ & $0.115 \pm 0.028$ \\
CIM & $0.17 \pm 0.05$ & $51.30 \pm 44.33$ & $3.13 \pm 1.79$ & & $0.21 \pm 0.01$ & $75.73 \pm 30.16$ & $3.27 \pm 0.5$ \\
ERY & $0.21 \pm 0.10$ & $43.70 \pm 28.49$ & $3.14 \pm 0.69$ & & $0.17 \pm 0.06$ & $42.42 \pm 17.01$ & $6.41 \pm 1.1$ \\
\hline
\end{tabular}

Each value is represented by mean $\pm \mathrm{SD}(\mathrm{n}=4)$. For each inhibitor, two reaction velocity-substrate concentrations curves were simultaneously analysed using a nonlinear least squares fitting program to estimate the kinetic parameter values including $V_{\max }, K_{m}$ and $K_{i}$.
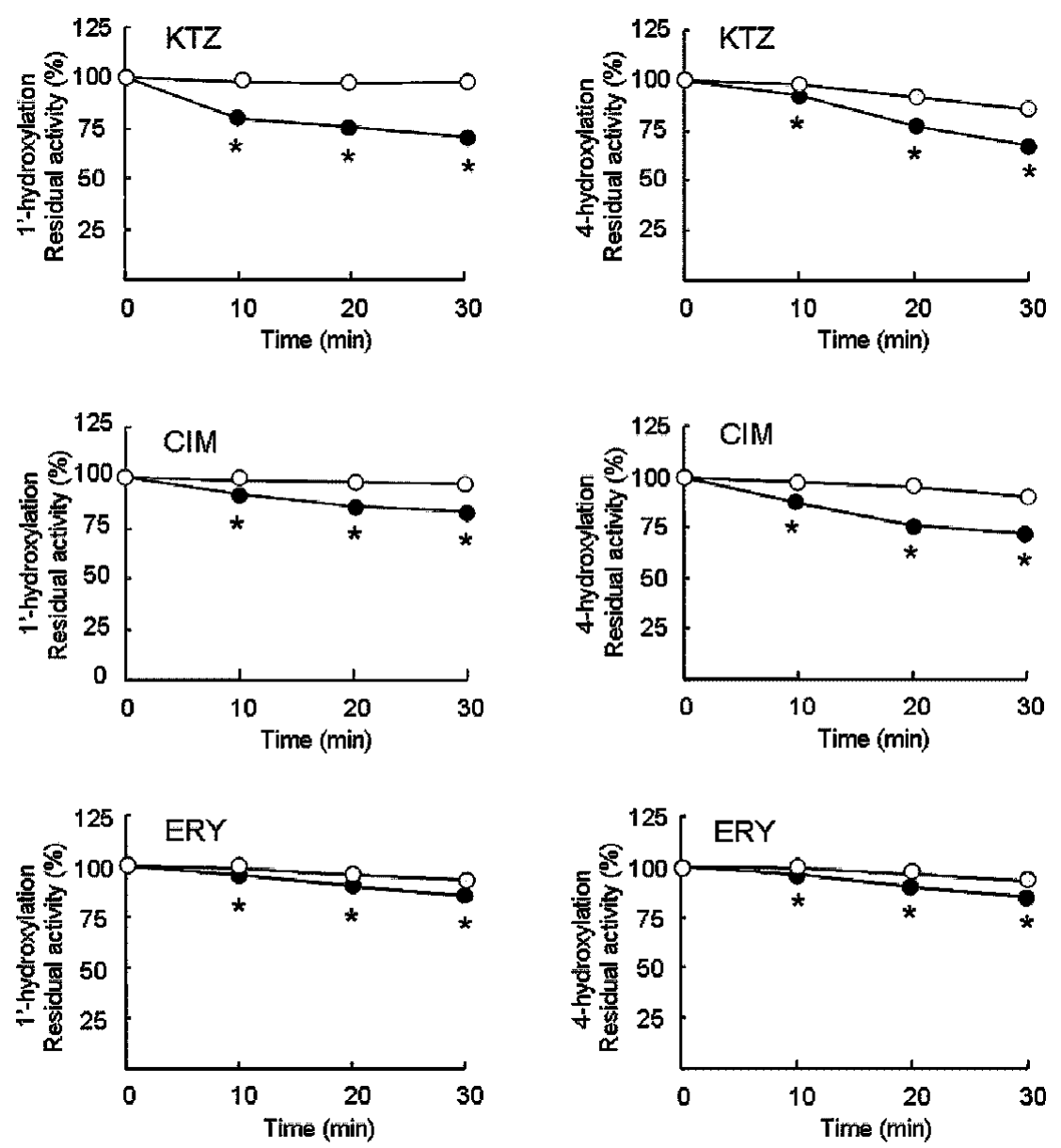

Fig. 3. Time-dependent inhibitory effects of ketoconazole (KTZ), cimetidine (CIM), and erythromycin (ERY) on 1'- and 4-hydroxylation of midazolam. Each point and vertical bar is represented by mean $\pm \mathrm{SD}$ from four microsomes. $\mathrm{X}$-axis shows incubation time of reaction mixture including microsomes, NADP and inhibitor before adding the substrate. Open circles represent reaction without inhibitors. Closed circles represent the reactions with KTZ (at $1 \mu \mathrm{M}$ for 1'hydroxylation and at $100 \mu \mathrm{M}$ for 4-hydroxylation), CIM (at $2.4 \mathrm{mM}$ ) or ERY (at $10.9 \mathrm{mM}$ ). The concentrations of midazolam were $123 \mu \mathrm{M}$. The inhibitory potency was expressed as percentage of catalytical activity in the absence of KTZ, CIM or ERY. Asterisk indicates significant difference in reactions between with and without inhibitors when $\mathrm{P}<0.05$. 


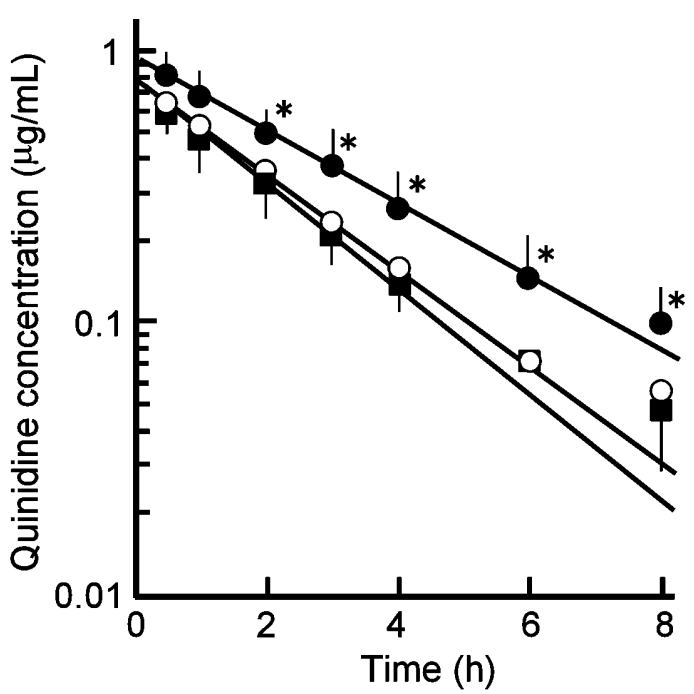

Fig. 4. Plasma concentrations against time curves of quinidine after intravenous injection at $2 \mathrm{mg} / \mathrm{kg}$ to cats obtained before and after oral treatment with ketoconazole (KTZ) or cimetidine (CIM) using a clinical prescription for a week. Each point and vertical bar is represented by mean \pm SD from five cats. Open circles represent plasma concentrations obtained 4 weeks before the treatment with inhibitors. Closed circles and closed squares represent those obtained after treatment with KTZ and CIM, respectively. In case of after treatment with inhibitors, quinidine was intravenously injected at $2 \mathrm{hr}$ after the final dose of KTZ or CIM. Asterisk indicates significant difference in concentrations between with and without treatment with inhibitors when $\mathrm{P}<0.05$.

cyclosporine in human [19] and dog [5, 14] and with midazolam in human $[3,7]$ and $\operatorname{dog}[10]$.

The extent of inhibitory effects of KTZ on MDZ1'H and MDZ4H seems to be quite different among animal species. The Ki values for MDZ1' $\mathrm{H}$ and MDZ4H have been reported to be $0.0037-0.18 \mu \mathrm{M}$ and $0.047 \mu \mathrm{M}$ in humans [25-27]. The values in dogs were $0.082 \mathrm{mM}$ for $\mathrm{MDZ1}{ }^{\prime} \mathrm{H}$ and 0.38 $\mu \mathrm{M}$ for $\mathrm{MDZ} 4 \mathrm{H}$, respectively [11]. Comparing with humans $K_{i}$ values, the values in cats were 16 to 700 and 2,000 fold higher for MDZ1'H and MDZ4H, respectively. On the other hand, the values were 34-fold and 300-fold higher, respectively, when compared with those of dogs. It is, therefore, suggested that KTZ could inhibit the hepatic CYP3A activities in cats at much less significantly than those in humans and dogs.

Since Yamano et al. [30] and Zhao et al. [31] reported mechanism-based inhibitory effects of itraconazole, CIM, and ERY on hepatic CYP3A activities in humans, we also examined this inhibitory mode in cats. As KTZ time-dependently inhibited MDZ1'H and MDZ4H with statistical significances, it may be suggested that KTZ inhibited these reactions by mechanism-based inhibition. This inhibitory effect might be substantial in feline patients, because the decrease in MDZ1'H was brought by a quite small concentration $(1 \mu \mathrm{M})$ of KTZ. Although CIM and ERY also decreased the reactions time-dependently, the decreases were small even though extremely high concentrations (millimolar range) were used.

In order to clarify whether the inhibitory effects of the CYP3A inhibitors, which we obtained from the in vitro studies, are observed in vivo, we also examined inhibitory effects of KTZ and CIM at clinically used dosage [20, 21] on QUN pharmacokinetics in cats. The multiple oral treatment of KTZ using the clinical dose $(10 \mathrm{mg} / \mathrm{kg}$, once daily) for one week significantly altered elimination profiles of QUN in cats (Table 2). Plasma QUN concentrations after a week treatment of KTZ were significantly higher than before treatment (Fig. 5). The elimination $t_{1 / 2}$ prolonged by 1.6-fold, and Cltot decreased by $35 \%$ (Table 2). In contrast, the multiple oral treatment of CIM using a clinical prescription $(10 \mathrm{mg} / \mathrm{kg}$, three times a day) for one week did not alter the elimination profiles of QUN (Table 2). In this study, we did not examine the effect of ERY on QUN pharmacokinetics in cats. However, it may be suggested that the drug also does not alter the QUN pharmacokinetics because of the following reasons: $K_{i}$ values of ERY was higher and its mechanism-based inhibition is lower than CIM.

In conclusion, the results in the present study suggest that

Table 2. Pharmacokinetic parameters of quinidine after intravenous injection at $2 \mathrm{mg} /$ $\mathrm{kg}$ with or without treatment with ketoconazole (KTZ) or cimetidine (CIM) in cat

\begin{tabular}{lccc}
\hline Parameters & Without treatment & KTZ treatment & CIM treatment \\
\hline$K_{e l}(/ \mathrm{hr})$ & $0.449 \pm 0.063^{\mathrm{a})}$ & $0.306 \pm 0.065^{\mathrm{a})}$ & $0.407 \pm 0.103$ \\
$A U C_{\text {0-inf }}(\mu \mathrm{g} \mathrm{h} / \mathrm{m} l)$ & $1.962 \pm 0.217^{\mathrm{b})}$ & $3.158 \pm 0.893^{\mathrm{b})}$ & $1.770 \pm 0.890$ \\
$\mathrm{~V}_{d}(\mathrm{~L} / \mathrm{kg})$ & $2.546 \pm 0.322$ & $2.237 \pm 0.493$ & $2.877 \pm 1.029$ \\
$t_{l / 2}(\mathrm{ht})$ & $1.500 \pm 0.352^{\mathrm{c})}$ & $2.353 \pm 0.539^{\mathrm{c})}$ & $1.780 \pm 0.405$ \\
$M R T(\mathrm{hr})$ & $2.411 \pm 0.264^{\mathrm{d})}$ & $3.364 \pm 0.780^{\mathrm{d})}$ & $2.627 \pm 0.602$ \\
$C l_{\text {tot }}(\mathrm{L} / \mathrm{h} / \mathrm{kg})$ & $1.031 \pm 0.129^{\mathrm{e})}$ & $0.673 \pm 0.181^{\mathrm{e})}$ & $1.399 \pm 0.716$ \\
\hline
\end{tabular}

Each value is represented by mean $\pm \mathrm{SD}(\mathrm{n}=5) . K_{e l}$, elimination rate constant; $A U C_{0 \text {-inf }}$, area under the curve from time $0 \mathrm{hr}$ to infinity; $V_{d}$, apparent Volume of distribution; $t_{1 / 2}$, elimination half-life; $M R T$, mean residence time $C l_{\text {tot }}$, total body clearance. KTZ or CIM was orally administered at $10 \mathrm{mg} / \mathrm{kg}$ body weight once a day and three times a day for a week, respectively. Quinidine was injected at 4 weeks before the start of dosing and $2 \mathrm{hr}$ after the final dosing of the inhibitors. Superscripts indicate significant differences between values with the same superscripts at $\mathrm{p}<0.05$. 
concomitant coadministration of KTZ with CYP3A substrates may result in drug-drug interaction in feline patients. Although it has been suggested that CYP3A activities are lower in female cats than male cats [22], the drug-drug interaction may be substantial even in male cats. Therefore, veterinary practitioners should pay much attention to potential adverse effects in feline patients, if they co-medicate KTZ with CYP3A substrates, such as cyclosporine. On the other hand, there may be much less possibilities of drug-drug interaction, when CYP3A substrates are given to feline patients treated with CIM and/or ERY.

\section{REFERENCES}

1. Benet, L. Z., Kroets, L. and Sheiner, L. B. 1996. Pharmacokinetics: The dynamics of drug absorption, distribution, and elimination. pp. 3-27. In: Googman \& Gilman's The Pharmacological Basis of Therapeutics, 9th ed., (Hardman, J. G. and Limbird, L. E. eds.), McGraw-Hill, New York.

2. Bradford, M. M. 1976. A rapid and sensitive method for the quantitation of microgram quantities of protein utilizing the principle of protein-dye binding. Anal. Biochem. 72: 248-254.

3. Chien, J. Y., Lucksiri, A., Ernest II, C. S., Gorski, C., Wrighton, S. A. and Hall, S. A. 2006. Stochastic predication of CYP3A-mediated inhibition of midazolam clearance by ketoconazole. Drug. Metab. Dispos. 34: 1208-1219.

4. Daigle, J. C., Hosgood, G., Foil, C. S. and Hunter, R. P. 2001. Effect of cimetidine on pharmacokinetics of orally administered cyclosporine in healthy dogs. Am. J. Vet. Res. 62: 10461050.

5. Dahlinger, J., Gregory, C. and Bea, J. 1998. Effect of ketoconazole on cyclosporine dose in healthy dogs. Vet. Surg. 27: 6468.

6. Edstein, M. D., Prasitthipayong, A., Sabchareon, A., Chongsuhajaisiddhi, T. and Webester, H. K. 1990. Simultaneous measurement of quinine and quinidine in human plasma, whole blood, and erythrocytes by high-performance liquid chromatography with fluorescence detection. Ther. Drug Monit. 12: 493-500.

7. Gibbs, M. A., Thummel, K. E., Shen, D. D. and Kunze, K. L. 1999. Inhibition of CYP3A in human intestinal and liver microsomes: comparison of Ki values and impact of CYP3A5 expression. Drug. Metab. Dispos. 27: 180-187.

8. Guengerich, F. P. 1999. Cytochrome P-450 3A4: regulation and role in drug metabolism. Annu. Rev. Pharmacol. Toxicol. 39: $1-17$.

9. Johnson, L. M., Lankford, S. M. and Bai, S. A. 1995. The influence of cimetidine on the pharmacokinetics of the enantiomers of verapamil in the dog during multiple oral dosing. $J$. Vet. Pharmacol. Therap. 18: 117-123.

10. Kuroha, M., Kayaba, H., Kishimoto, S., Khalil, W. F., Shimoda, M. and Kokue, E. 2002. Effect of oral ketoconazole on first-pass effect of nifedipine after oral administration in dogs. J. Pharm. Sci. 91: 868-873.

11. Kuroha, M., Azumano, A., Kuze, Y., Shimoda, M. and Kokue, E. 2002. Effect of multiple dosing of Ketoconazole on pharmacokinetics of midazolam, a cytochrome P-450 3A substrate in beagle dogs. Drug. Metab. Dispos. 30: 63-68.

12. Kuroha, M., Kuze, Y., Shimoda, M. and Kokue, E. 2002. In vitro characterization of the inhibitory effects of Ketoconazole on metabolic activities of cytochrome P-450 in canine hepatic microsomes. Am. J. Vet. Res. 63: 900-905.
13. Kuroha, M., Shirai, Y. and Shimoda, M. 2004. Multiple oral dosing of Ketoconazole influences pharmacokinetics of quinidine after intravenous and oral administration in beagle dogs. $J$. Vet. Pharmacol. Therap. 27: 355-359.

14. Myre, S. A., Schoeder, T. J., Grund, V. R., Wandstrat, T. L., Nicelv, P. G., Pesce, A. J. and First, M. R. 1991. Critical ketoconazole dosage range for cyclosporine clearance inhibition in the dog. Pharmacology 43: 233-241.

15. Martinez, C., Albet, C., Agundez, J. A., Herrero, E., Carrillo, J. A., Marquez, M., Benitez, J. and Ortiz, J. A. 1999. Comparative in vitro and in vivo inhibition of cytochrome $\mathrm{P} 450$ CYP1A2, CYP2D6, and CYP3A by H2-receptor antagonists. Clin. Parmacol. Ther. 65: 369-376.

16. McAnulty, J. F. and Lensmeyer G. L. 1999. The effects of ketoconazole on the pharmacokinetics of cyclosporine A in cats. Vet. Surg. 28: 448-455.

17. Newton, D. J., Wang, R. W. and Lu, A. Y. 1995. Cytochrome P450 inhibitors. Evaluation of specificities in the in vitro metabolism of therapeutic agents by human liver microsomes. Drug. Metab. Dispos. 23: 154-158.

18. Omura, T. and Sato, R. 1964. The carbon monoxide binding pigment of liver microsomes. J. Biol. Chem. 239: 2370-2378.

19. Pichard, L., Fabre, I., Fabre, G., Domergue, J., Saint Aubert, B., Mourad, G. and Maurel, P. 1990. Cyclosporine A drug interactions. Screening for inducers and inhibitors of cytochrome P-450 (Cyclosporin A oxidase) in primary cultures of human hepatocytes and in liver microsomes. Drug Metab. Dispos. 18: 595-606.

20. Plumb, D. C. 1999. Ketoconazole. pp. 365-369. In: Veterinay Drug Handbook, 3rd ed. (Plumb, D. C. ed.), Iowa State Press, Ames.

21. Plumb, D. C. 1999. Cimetidine. pp. 134-137. In: Veterinay Drug Handbook, 3rd ed. (Plumb, D. C. ed.), Iowa State Press, Ames.

22. Shah, S. S., Sanda, S., Regmi, N. L., Sasaki, K. and Shimoda, M. 2007. Characterization of cytochrome P450-mediated drug metabolism in cats. J. Vet. Pharmacol. Therap. 30: 422-428

23. Trepanier, L. A. 2006. Cytochrome P450 and its role in veterinary drug interaction. Vet. Clin. North Am. Small Anim. Pract. 36: $975-985$.

24. Van der Hoeven, T. A. and Coon, M. J. 1974. Preparation and properties of partially purified cytochrome P-450 and reduced nicotinamide adenine dinucleotide phosphate-cytochrome P450 reductase from rabbit liver microsomes. J. Biol. Chem. 249: $6302-6310$.

25. Von Moltke L. L., Greenblatt D. J., Schmider J., Duan S. X., Wright C. E., Harmatz, J. S. and Shader, R. I. 1996. Midazolam hydroxylation by human liver microsomes in vitro: Inhibition by fluoxetine, norfluoxetine, and by azole antifungal agents. $J$. Clin. Pharmacol. 36: 783-791.

26. Wang, J. S., Wen, X., Backman, J. T., Taavitsainen, P., Neuvonen, P. J. and Kivisto, K. T. 1999. Midazolam alpha-hydroxylation by human liver microsomes in vitro: Inhibition by calcium channel blockers, itraconazole and ketoconazole. Pharmacol. Toxicol. 85: 157-161.

27. Wrighton, S. A., Vandenbranden, M., Stevens, J. C., Shipley, L. A., Ring, B. J., Rettie, A. E. and Cashman, J. R. 1993. In vitro methods for assessing human hepatic drug metabolism: their use in drug development. Drug Metab. Rev. 25: 453-484.

28. Wrighton, S. A. and Ring, B. J. 1994. Inhibition of human CYP3A catalyzed 1'-hydroxymidazolam formation by ketoconazole, nifedipine, erythromycin, cimitidine, and nizatidine. Pharm. Res. 11: 921-924. 
29. Yamaoka, K., Tanigawara, Y., Nakagawa, T. and Uno, T. 1981. A pharmacokinetic analysis program (MULTI) for microcomputer. J. Pharmacobio-Dyn. 4: 879-885.

30. Yamano, K., Yamamoto, K., Katashima, M, Kotaki, H., Takedomi, S., Matsuo, H., Ohtani, H., Sawada, Y. and Iga, T. 2001. Predication of midazolam- CYP3A inhibitors interaction in the human liver from in vivo/in vitro absorption, distribution, and metabolism data. Drug Metab. Dispos. 29: 443-452.

31. Zhao, P., Kunze, K. L. and Lee, C. A. 2005. Evaluation of time-dependent inactivation of CYP3A in cryopreserved human hepatocytes. Drug Metab. Dispos. 33: 853-861.

32. Zuber, R., Anzenbacherova, E. and Anzenbacher, P. 2002. Cytochrome P450 and experimental models of drug metabolism. J. Cellular Mol. Med. 6: 189-198. 